\title{
Gauge dependence of the critical dynamics at the superconducting phase transition
}

\author{
M.Dudka ${ }^{1,2}$, R.Folk², G.Moser ${ }^{3}$ \\ 1 Institute for Condensed Matter Physics of the National Academy of Sciences of Ukraine, 79011 Lviv, Ukraine \\ 2 Institut für Theoretische Physik, Johannes Kepler Universität Linz, A-4040 Linz, Austria \\ 3 Institut für Physik und Biophysik, Universität Salzburg, A-5020 Salzburg, Austria
}

Received December 27, 2006, in final form May 7, 2007

\begin{abstract}
The critical dynamics of superconductors in the charged regime is reconsidered within field-theory. For the dynamics, the Ginzburg-Landau model with complex order parameter coupled to the gauge field suggested earlier [Lannert et al. Phys. Rev. Lett. 92, 097004 (2004)] is used. Assuming relaxational dynamics for both quantities, the renormalization group functions within one loop approximation are recalculated for different choices of the gauge. A gauge independent result for the divergence of the measurable electric conductivity is obtained only at the weak scaling fixed point unstable in one loop order where the time scales of the order parameter and the gauge field are different.
\end{abstract}

Key words: critical dynamics, superconducting transition

PACS: $05.70 . J k, 64.60 . A k, 64.60 . H t, 74.20 .-z$

\section{Introduction}

The nature of the static phase transition in superconductors was an open question for decades, since due to the large correlation length of the available superconducting materials the effect of critical fluctuations was hard to observe in the vicinity of the critical temperature $T_{\mathrm{c}}$. Moreover, it was unclear whether the phase transition was of first or second order and in case it was of second order, to which universality class the transition belonged. The appearance of high- $T_{\mathrm{c}}$ superconducting compounds with short correlation lengths [1] made the critical region of superconductors experimentally accessible. In turn, this leads to a comparison of the experiment with analytical results in order to establish the critical properties of superconductors and their universality class.

From the theoretical point of view the static critical properties are now well understandable. The theoretical model for the description of static critical properties was formulated in [2] and contains, besides the two component $(n=2)$ order parameter (OP) and its fourth order interaction term, a minimal coupling to a gauge field (GF) due to the charged character of the OP (macroscopic wave function of the Cooper pair). The coupling of the OP to the GF introduces an essential difference to the superfluid phase transition, where the OP is uncharged.

While for type-I superconductors the fluctuation effects are weak and a mean-field analysis can be applied, the situation with type-II superconductors is more complicated since here fluctuations of the OP cannot be neglected. The first renormalization group (RG) analysis in one-loop approximation [2] leads to the conclusion that a stable fixed point (FP) and thus a second order phase transition only exist for OP dimensions $n$ larger than 365.9. For OP dimension $n=2$ no stable FP was found and the runaway solution was interpreted as a weak first order phase transition. A two loop order calculation of the renormalization group equations within the field theory indicated the possibility of a continuous phase transition for $n=2[3]$ if certain resummations are performed. This was investigated further and led to the following picture [4]: There are four FPs, two uncharged ones known from the standard Ginzburg-Landau-Wilson (GLW) model and two charged ones. Depending on the initial (background) conditions for the flow of the fourth order coupling and the coupling to the charge (defining the value of the Ginzburg parameter $\kappa$ ), a runaway flow 
is obtained or the flow reaches the stable charged FP. The other charged FP is reached starting at the separatrix (defined by $\kappa=\kappa_{\mathrm{c}}$ ) separating the attraction region of the charged FP from the runaway region. Physically this FP reached at the separatrix describes tricritical behavior indicating that a tricritical point separates the first order transition of the superconductors of type I from the second order transition for superconductors of type II. The results of duality arguments [5] and Monte Carlo calculations [6] are in agreement with this picture, which is also supported by experiment $[7,8]$.

An important question in calculating critical properties like the critical exponents is their dependence on the gauge used for the GF. Physically observable quantities should be independent of the specific gauge used. This has been shown in one loop order [9]. However non-observable quantities might depend on the gauge. Thus the critical exponents $\nu$ or $\alpha$ of the penetration length $\lambda$ (proportional to the correlation length $\xi$ of the OP correlation function at the charged $\mathrm{FP}$ ) and the specific heat, respectively, should be independent of gauge, whereas the critical exponent of the static OP correlation function turns out to be gauge dependent [10].

The issue of dynamical critical properties was less studied. Experimental investigations give no consistent picture of the dynamical critical exponent $z$ (for references see [11]) obtained from measurements of the electrical conductivity. The values of the dynamical critical exponents found vary between 1.5 and 2.3 .

Theoretical predictions of $z$ are mainly based on the results of the known universality classes (model A, C, E or model F, for a review see [12]). Model A is the simplest model assuming a relaxation equation for the $\mathrm{OP}$ without coupling to other conserved densities, whereas model $\mathrm{C}$ contains a coupling to a diffusion equation for a conserved density (only relevant if the specific heat is diverging). Models $\mathrm{E}$ and $\mathrm{F}$ define the universality class of the critical dynamics of the superfluid transition in ${ }^{4}$ He. Dynamical equations from the vortex-loop model [13,14] indicate a relation to that universality class. So far no systematic derivation of dynamical equations with mode coupling terms derived from Poisson bracket relations using for instance methods described in [15] has been performed.

Monte Carlo simulations [13] in the limiting cases of large and small values of the Ginzburg parameter established the values of the dynamical exponent of 2.7 and 1.5, respectively. Using the vortex model [11] for superconducting transition, the dynamical critical exponent has been analytically calculated in the two limits mentioned above. The resulting exponents were $5 / 2$ and $3 / 2$, respectively, and they were related to the dynamical exponents obtained in simulations. A discussion [16] arose concerning the cause of the deviation of the Monte Carlo results from the expected value of model A, which would lead to a dynamical exponent of about 2. Recently in [14] for extreme type II superconductors, where the uncharged FP describes the static behavior, it was argued, using scaling and duality arguments, that $z=3 / 2$ exactly.

A concrete dynamical model defined by equations of motion for the OP and the GF has been presented in [17]. Both equations are of relaxational character and the essential parameter is the ratio $w=\Gamma_{\psi} / \Gamma_{\mathrm{A}}$ of the kinetic coefficients $\Gamma_{\psi}$ of the OP and $\Gamma_{\mathrm{A}}$ of the GF. A dynamical FP, $w^{\star}$ finite, implying strong scaling with a common dynamical critical exponent $z$ for the OP and the GF in the Feynman gauge (one adds a quadratic term in the divergence of the GF to the static functional) has been found in one loop order. However, the question of the gauge dependence of the dynamical exponents was not addressed in [17].

Another important issue not considered so far in the discussion of the dynamical critical behavior concerns the time scales entering the problem. One has to discriminate between the time scale for the OP and its dynamical critical exponent $z_{\psi}$ and the time scale of the GF and its dynamical critical exponent $z_{\mathrm{A}}$. They are defined by the characteristic frequencies of the dynamical correlation functions for the $\mathrm{OP}$ and the GF, respectively

$$
\omega_{\psi} \sim k^{z_{\psi}} g_{\psi}(k \xi), \quad \omega_{\mathrm{A}} \sim k^{z_{\mathrm{A}}} g_{\mathrm{A}}(k \xi)
$$

$g_{\psi}$ and $g_{\mathrm{A}}$ are scaling functions which are finite and nonzero at the superfluid transition $T_{\mathrm{c}}$.

The dynamical critical exponents are different [18] if weak scaling holds. Otherwise one says the dynamical correlation functions obey strong scaling. So far in the discussion the latter case was 
assumed. Even if for the uncharged FP, the dynamics would be in the universality class of model $\mathrm{E}$, it is the weak scaling $\mathrm{FP}$ which is stable [19].

Only one of these dynamical exponents is observable, namely $z_{\mathrm{A}}$ entering the frequency dependent diverging electric conductivity

$$
\sigma(\xi, k=0, \omega) \sim \xi^{\left(z_{\mathrm{A}}-2+\eta_{\mathrm{A}}\right)},
$$

$\eta_{\mathrm{A}}=4-d$ is the anomalous dimension of the GF independent of the gauge [20]. This implies that the dynamical critical exponent $z_{\mathrm{A}}$ has to be gauge independent. No such condition holds for $z_{\psi}$. Therefore, if it turns out that $z_{\psi}$ is gauge dependent, strong scaling cannot hold.

In this paper we apply the field-theoretical RG approach in the minimal subtraction scheme and dimensional regularization to the dynamical model of [17] in order to investigate the gauge dependence of the critical dynamics. It turns out that the dynamical exponent $z_{\psi}$ of the OP is gauge dependent and a gauge independent value for $z_{\mathrm{A}}$ is only obtained in the weak scaling $\mathrm{FP} w^{\star} \rightarrow \infty$, which, however, has been found unstable. Therefore, one has to question the physical relevance of the one loop result. This goes together with the problems in statics of the Ginzburg-Landau model at the one loop order level.

\section{Model}

Static critical properties of charged superconductors in $d$ dimensional space are described by the Abelian Higgs model with the static functional [2]:

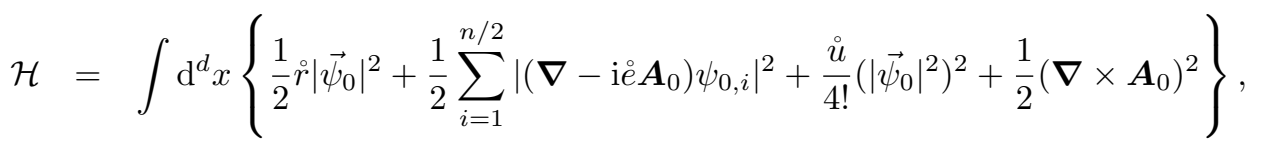

with the complex $n$-component $\mathrm{OP} \vec{\psi}_{0}$ (generalized to $n / 2$-components, the superconductor being the case $n=2$ ) and the $d$-dimensional GF $\boldsymbol{A}_{\mathbf{0}}$. The bare parameter $\stackrel{\circ}{r}$ is proportional to the distance

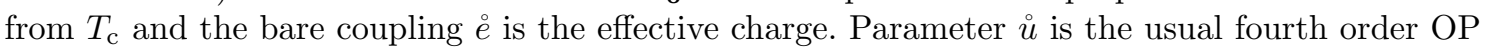
coupling. In all subsequent calculations we add the contribution

$$
\mathcal{H}=\frac{1}{2 \varsigma}\left(\nabla \cdot \boldsymbol{A}_{0}\right),
$$

to the static functional which allows us to choose the gauge. In the limit $\stackrel{\circ}{\rightarrow} 0$, the Landau gauge is recovered, while for a system with $\stackrel{\circ}{\rightarrow} 1$, the Feynman gauge is achieved.

The OP and the GF are nonconserved quantities. Thus, their dynamical behavior may be described by relaxation equations. Such a set of equations of motion has been suggested [17]:

$$
\begin{aligned}
\frac{\partial \psi_{0, i}}{\partial t} & =-2 \stackrel{\circ}{\Gamma}_{\psi} \frac{\delta \mathcal{H}}{\delta \psi_{0, i}^{+}}+\theta_{i} ; \\
\frac{\partial \psi_{0, i}^{+}}{\partial t} & =-2 \stackrel{\circ}{\Gamma}_{\psi} \frac{\delta \mathcal{H}}{\delta \psi_{0, i}}+\theta_{i}^{+} ; \\
\frac{\partial A_{0, \alpha}}{\partial t} & =-\stackrel{\circ}{\Gamma}_{\mathrm{A}} \frac{\delta \mathcal{H}}{\delta A_{0, \alpha}}+\theta_{\alpha} .
\end{aligned}
$$

The OP relaxes with the kinetic coefficient $\Gamma_{\psi}$ while the GF relaxes with the kinetic coefficient $\Gamma_{\mathrm{A}}$. The stochastic forces in (5) are related to the kinetic coefficients and satisfy the relations:

$$
\begin{array}{rlr}
\left\langle\theta_{i}(\mathbf{x}, t) \theta_{j}^{+}\left(\mathbf{x}^{\prime}, t^{\prime}\right)\right\rangle & =4 \stackrel{\circ}{\Gamma}_{\psi} \delta\left(\mathbf{x}-\mathbf{x}^{\prime}\right) \delta\left(t-t^{\prime}\right) \delta_{i j}, & \left\langle\theta_{i}(\mathbf{x}, t)\right\rangle=0, \\
\left\langle\theta_{\alpha}(\mathbf{x}, t) \theta_{\beta}\left(\mathbf{x}^{\prime}, t^{\prime}\right)\right\rangle & =2 \stackrel{\circ}{\Gamma}_{\mathrm{A}} \delta\left(\mathbf{x}-\mathbf{x}^{\prime}\right) \delta\left(t-t^{\prime}\right) \delta_{\alpha \beta}, & \left\langle\theta_{\alpha}(\mathbf{x}, t)\right\rangle=0 .
\end{array}
$$

The indices $i, j$ adopt the values $1, \ldots, n / 2$, while the indices $\alpha, \beta$ adopt the values $1, \ldots, d$. 
As it was pointed out in [17] the equation for the GF in (5) can be derived from Maxwell's equations in their low-frequency form. In this case the inverse transverse coefficient $\stackrel{\circ}{\Gamma}_{\mathrm{A}}^{-1}$ for the GF can be identified with the bare normal electrical conductivity.

We study this critical dynamics by using the Bausch-Janssen-Wagner approach [21] of dynamical field-theoretical RG. In this approach, the critical behavior is considered based on the long-distance and long-time properties of the Lagrangian incorporating the features defined by the dynamical equations of the model:

$$
\begin{aligned}
\mathcal{L}= & \int \mathrm{d}^{d} x \mathrm{~d} t \sum_{i=1}^{n / 2} \tilde{\psi}_{0, i}^{+}\left[\frac{\partial \psi_{0, i}}{\partial t}+2 \stackrel{\circ}{\Gamma}_{\psi} \frac{\delta \mathcal{H}}{\delta \psi_{0, i}^{+}}-2 \Gamma_{\psi} \tilde{\psi}_{0, i}^{+}\right]+\sum_{i=1}^{n / 2} \tilde{\psi}_{0, i}\left[\frac{\partial \psi_{0, i}^{+}}{\partial t}+2 \stackrel{\circ}{\Gamma}_{\psi} \frac{\delta \mathcal{H}}{\delta \psi_{0, i}}-2 \Gamma_{\psi} \tilde{\psi}_{0, i}\right] \\
& +\sum_{\alpha=1}^{d} \tilde{A}_{0, \alpha}\left[\frac{\partial A_{0, \alpha}}{\partial t}+\stackrel{\circ}{\Gamma}_{\mathrm{A}} \frac{\delta \mathcal{H}}{\delta A_{0, \alpha}}-\stackrel{\circ}{\Gamma}_{\mathrm{A}} \tilde{A}_{0, \alpha}\right] .
\end{aligned}
$$

Here, in order to keep the powers in the interaction terms low an auxiliary OP density $\overrightarrow{\tilde{\psi}}_{0}$ and an auxiliary GF $\tilde{\boldsymbol{A}}$ have been introduced. The dynamical equations defined by (3)-(6) are then described by the unrenormalized Lagrangian (7), which we separate $\mathcal{L}=\mathcal{L}_{0}+\mathcal{L}_{1}$ into a Gaussian part

$$
\begin{aligned}
\mathcal{L}_{0}= & \int \mathrm{d}^{d} x \mathrm{~d} t\left[-4 \stackrel{\circ}{\Gamma} \sum_{i=1}^{n / 2} \tilde{\psi}_{0, i}^{+} \tilde{\psi}_{0, i}-\stackrel{\circ}{\Gamma}_{\mathrm{A}} \tilde{\boldsymbol{A}}_{0}^{2}+\sum_{i=1}^{n / 2} \tilde{\psi}_{0, i}^{+}\left(\frac{\partial}{\partial t}+\stackrel{\circ}{\Gamma}_{\psi}(\stackrel{\circ}{r}-\Delta)\right) \psi_{0, i}\right. \\
& \left.+\sum_{i=1}^{n / 2} \tilde{\psi}_{0, i}\left(\frac{\partial}{\partial t}+\stackrel{\circ}{\Gamma}_{\psi}(\stackrel{\circ}{r}-\Delta)\right) \psi_{0, i}^{+}+\tilde{\boldsymbol{A}}_{0} \cdot\left(\frac{\partial \boldsymbol{A}_{0}}{\partial t}+\stackrel{\circ}{\Gamma}_{\mathrm{A}}\left(\boldsymbol{\nabla}\left(1-\frac{1}{\varsigma}\right)\left(\boldsymbol{\nabla} \cdot \boldsymbol{A}_{0}\right)-\Delta \boldsymbol{A}_{0}\right)\right)\right],
\end{aligned}
$$

and an interaction part

$$
\begin{aligned}
\mathcal{L}_{1}= & \int \mathrm{d}^{d} x \mathrm{~d} t\left[\stackrel{\circ}{\Gamma}_{\psi} \frac{\stackrel{i}{u}}{3 !}|\psi|^{2} \sum_{i=1}^{n / 2}\left(\tilde{\psi}_{0, i}^{+} \psi_{0, i}+\tilde{\psi}_{0, i} \psi_{0, i}^{+}\right)+2 \stackrel{\circ}{\Gamma}_{\psi} \mathrm{i} \stackrel{\circ}{ } \boldsymbol{A}_{0} \cdot \sum_{i=1}^{n / 2}\left(\tilde{\psi}_{0, i}^{+} \boldsymbol{\nabla} \psi_{0, i}-\tilde{\psi}_{0, i} \boldsymbol{\nabla} \psi_{0, i}^{+}\right)\right. \\
& +\stackrel{\circ}{\Gamma}_{\psi} \mathrm{i} \mathrm{i}\left(\boldsymbol{\nabla} \cdot \boldsymbol{A}_{0}\right) \sum_{i=1}^{n / 2}\left(\tilde{\psi}_{0, i}^{+} \psi_{0, i}-\tilde{\psi}_{0, i} \psi_{0, i}^{+}\right)+\stackrel{\circ}{\Gamma}_{\mathrm{A}} \frac{1}{2} \mathrm{i} \stackrel{\circ}{\tilde{\boldsymbol{A}}_{0}} \cdot \sum_{i=1}^{n / 2}\left(\psi_{0, i} \boldsymbol{\nabla} \psi_{0, i}^{+}-\psi_{0, i}^{+} \boldsymbol{\nabla} \psi_{0, i}\right) \\
& \left.+\stackrel{\circ}{\Gamma}_{\psi} \stackrel{\circ}{ }^{2} \boldsymbol{A}_{0}^{2} \sum_{i=1}^{n / 2}\left(\tilde{\psi}_{0, i}^{+} \psi_{0, i}+\tilde{\psi}_{0, i} \psi_{0, i}^{+}\right)+\stackrel{\circ}{\Gamma}_{\mathrm{A}} \stackrel{\circ}{2}^{2} \tilde{\boldsymbol{A}}_{0} \cdot \boldsymbol{A}_{0}|\psi|^{2}\right] .
\end{aligned}
$$

The Lagrangian functional serves to calculate the dynamical vertex, response and correlation functions in perturbation theory using the weight function $W \sim e^{-\mathcal{L}}$. For instance, the correlation function for an order parameter is calculated as the average $\left\langle\psi \tilde{\psi}^{+}\right\rangle$. The description of dynamical vertex functions structure is given in the next section.

\section{Perturbative expansion and renormalization}

\subsection{Vertex functions}

In order to proceed, the vertex functions for the OP and the GF are calculated in one loop expansion. We keep the general structure of the two point vertex functions [12] and separate the static contributions. The calculation leads to the following general form of the OP vertex function

$$
\stackrel{\circ}{\Gamma}_{\psi \tilde{\psi}^{+}}=-\mathrm{i} \omega \stackrel{\circ}{\Omega}_{\psi \tilde{\psi}^{+}}+2 \stackrel{\circ}{\Gamma}_{\psi} \stackrel{\circ}{\Gamma}_{\psi \psi^{+}}^{\mathrm{st}}
$$


In one loop order, the dynamical OP function $\stackrel{\Omega}{\Omega}_{\psi \tilde{\psi}^{+}}$reads

$$
\begin{aligned}
& \stackrel{\circ}{\Omega}_{\psi \tilde{\psi}^{+}}=1+4 \stackrel{\circ}{e}^{2} \stackrel{\circ}{\Gamma}_{\psi} \int \frac{1}{\left(r+\left(k+k^{\prime}\right)^{2}\right) k^{\prime 2}\left(-\mathrm{i} \omega+\stackrel{\circ}{\Gamma}_{\psi}\left(\stackrel{\circ}{r}+\left(k+k^{\prime}\right)^{2}\right)+\stackrel{\circ}{\Gamma}_{\mathrm{A}} k^{\prime 2}\right)}\left(k^{2}-\frac{\left(k k^{\prime}\right)^{2}}{k^{\prime 2}}\right) \\
& +\stackrel{\circ}{e}^{2} \stackrel{\circ}{\Gamma}_{\psi} \stackrel{\circ}{\varsigma} \int \frac{1}{\left(\stackrel{\circ}{r}+\left(k+k^{\prime}\right)^{2}\right) k^{\prime 2}\left(-\mathrm{i} \omega \varsigma^{\circ}+\stackrel{\circ}{\Gamma}^{\circ}\left(r+\left(k+k^{\prime}\right)^{2}\right)+\stackrel{\circ}{\Gamma}_{\mathrm{A}} k^{\prime 2}\right)}\left(\frac{\left(\left(2 k+k^{\prime}\right) k^{\prime}\right)^{2}}{k^{\prime 2}}\right),
\end{aligned}
$$

whereas the static OP vertex function is given by

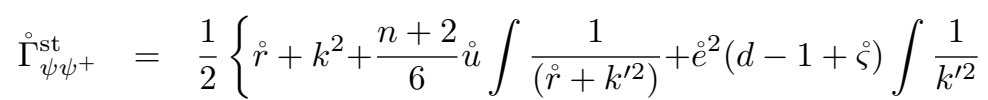

$$
\begin{aligned}
& \left.-4 e^{2} \int \frac{1}{\left(r+\left(k+k^{\prime}\right)^{2}\right) k^{\prime 2}}\left(k^{2}-\frac{\left(k k^{\prime}\right)^{2}}{k^{\prime 2}}+\stackrel{\circ}{\varsigma} \frac{\left(\left(2 k+k^{\prime}\right) k^{\prime}\right)^{2}}{k^{\prime 2}}\right)\right\} \text {. }
\end{aligned}
$$

The same general structure holds for the GF vertex function

$$
\stackrel{\circ}{\Gamma}_{A \tilde{A}}^{\alpha \beta}=-\mathrm{i} \omega \stackrel{\circ}{\Omega}_{A \tilde{A}}^{\alpha \beta}+\stackrel{\circ}{\Gamma}_{\mathrm{A}} \stackrel{\circ}{\Gamma}_{A A}^{\mathrm{st} \alpha \beta},
$$

with the one loop expression

$$
\stackrel{\circ}{\Omega}_{A \tilde{A}}^{\alpha \beta}=\delta^{\alpha \beta}+\stackrel{\circ}{\Gamma}_{\mathrm{A}} \frac{n}{2} \int \frac{\left(k+2 k^{\prime}\right)^{\alpha}\left(k+2 k^{\prime}\right)^{\beta}}{\left(\stackrel{\circ}{r}+k^{\prime 2}\right)\left(\stackrel{\circ}{r}+\left(k+k^{\prime}\right)^{2}\right)\left[-i \omega+\stackrel{\circ}{\Gamma}_{\psi}\left(\stackrel{\circ}{r}+k^{\prime 2}\right)+\stackrel{\circ}{\Gamma}_{\psi}\left(r+\left(k+k^{\prime}\right)^{2}\right)\right]},
$$

for the dynamical GF function and

$$
\stackrel{\circ}{\Gamma}_{A A}^{\text {st } \alpha \beta}=k^{2}\left(\delta^{\alpha \beta}-\frac{k^{\alpha} k^{\beta}}{k^{2}}+\frac{1}{\varsigma} \frac{k^{\alpha} k^{\beta}}{k^{2}}\right)+n \check{e}^{2} \int \frac{1}{\grave{r}+k^{\prime 2}}\left(\delta_{\alpha \beta}-\frac{1}{2} \frac{\left(k+2 k^{\prime}\right)^{\alpha}\left(k+2 k^{\prime}\right)^{\beta}}{\dot{r}+\left(k+k^{\prime}\right)^{2}}\right),
$$

for the GF static vertex function.

In an Appendix we present the diagrams for the one loop calculation of the corresponding vertex functions.

The renormalization procedure of vertex functions given above is described in the next subsection.

\subsection{Renormalization and field theoretic functions}

In order to get finite results we perform renormalization within the minimal subtraction scheme introducing renormalization factors leading to the renormalized parameters. The renormalization factors of the GLW part of the static functional are introduced as usual

$$
\psi_{0, i}=Z_{\psi}^{1 / 2} \psi_{i}, \quad \stackrel{\circ}{r}-\stackrel{\circ}{\mathrm{c}}=Z_{\psi}^{-1} Z_{r} r, \quad \stackrel{\imath}{u}=\kappa^{\varepsilon} \mathcal{A}_{d}^{-1} Z_{u} Z_{\psi}^{-2} u,
$$

where the shift of the phase transition temperature $\dot{r}_{\mathrm{c}}$ has been taken into account. Parameter $\kappa$ represents the free wave vector scale and $\varepsilon=4-d$. We also introduced the usual geometric factor

$$
\mathcal{A}_{d}=\Gamma\left(1-\frac{\epsilon}{2}\right) \Gamma\left(1+\frac{\epsilon}{2}\right) \frac{\Omega_{d}}{(2 \pi)^{d}},
$$

where $\Omega_{d}$ is the surface of the $d$-dimensional unit sphere. The additional renormalization factors due to the presence of the GF and its coupling to the OP are introduced as follows:

$$
A_{0, \alpha}=Z_{\mathrm{A}}^{1 / 2} A_{\alpha}, \quad \AA^{2}=\kappa^{\varepsilon} \mathcal{A}_{d}^{-1} Z_{e^{2}} Z_{\psi}^{-1} Z_{\mathrm{A}}^{-1} e^{2}, \quad \stackrel{\varsigma}{=} Z_{\varsigma}^{-1} Z_{\mathrm{A}} \varsigma .
$$

From Ward identities one derives the relations [9]

$$
Z_{e^{2}}=Z_{\psi}, \quad Z_{\varsigma}=1,
$$


which show that only one additional (for the GF) renormalization constant with respect to the GLW model appears.

In dynamics, two additional renormalization factors are needed which are as follows:

$$
\tilde{\psi}_{0, i}=Z_{\tilde{\psi}}^{1 / 2} \tilde{\psi}_{i}, \quad \tilde{A}_{0, \alpha}=Z_{\tilde{\mathrm{A}}}^{1 / 2} \tilde{A}_{\alpha} .
$$

For the kinetic coefficients no new factors are necessary

$$
\begin{aligned}
& \stackrel{\circ}{\Gamma}_{\psi}=Z_{\Gamma_{\psi}} \Gamma_{\psi}=Z_{\psi}^{1 / 2} Z_{\tilde{\psi}}^{-1 / 2} \Gamma_{\psi}, \\
& \stackrel{\circ}{\Gamma}_{\mathrm{A}}=Z_{\Gamma_{\mathrm{A}}} \Gamma_{\mathrm{A}}=Z_{\mathrm{A}}^{1 / 2} Z_{\tilde{\mathrm{A}}}^{-1 / 2} \Gamma_{\mathrm{A}},
\end{aligned}
$$

where the second relations are due to the structure of the vertex functions (see (10) and (13)).

The renormalization factors calculated in one loop order for the statics are as follows:

$$
\begin{gathered}
Z_{\psi}=1+(3-\varsigma) \frac{e^{2}}{\varepsilon}, \quad Z_{\mathrm{A}}=1-n \frac{e^{2}}{6 \varepsilon}, \\
Z_{r}=1+\frac{n+2}{6} \frac{u}{\varepsilon}-\varsigma \frac{e^{2}}{\varepsilon}, \quad Z_{u}=1+\frac{n+8}{6} \frac{u}{\varepsilon}+\frac{18}{u} \frac{e^{4}}{\varepsilon}-2 \varsigma \frac{e^{2}}{\varepsilon},
\end{gathered}
$$

as presented in [9]. The dynamical renormalization factors are correspondingly:

$$
\begin{aligned}
& Z_{\tilde{\psi}}=1-(3-\varsigma) \frac{e^{2}}{\varepsilon}-2 \frac{e^{2} \varsigma}{\varepsilon} \frac{w}{1+w}, \\
& Z_{\tilde{\mathrm{A}}}=1+n \frac{e^{2}}{2 \varepsilon}\left(\frac{1}{3}-\frac{1}{w}\right),
\end{aligned}
$$

where we have introduced the time scale ratio

$$
w=\frac{\Gamma_{\psi}}{\Gamma_{\mathrm{A}}},
$$

whose FP value $w^{\star}$ determines whether strong $\left(w^{\star} \neq 0\right.$ or $\left.\infty\right)$ or weak scaling $\left(w^{\star}=0\right.$ or $\left.\infty\right)$ holds.

From the $Z$-factors one obtains the $\zeta$-functions leading to the $\beta$-functions which determine the FPs. The critical exponents describing the critical properties are then expressed by the values of the $\zeta$-functions at stable FPs. We use the unified definition

$$
\zeta_{a}(\{\alpha\}, \varsigma)=-\frac{\mathrm{d} \ln Z_{a}}{\mathrm{~d} \ln \kappa}
$$

for all $\zeta$-functions, where $a$ denotes either any model parameter $\{\alpha\}=\left\{u, e^{2}, \Gamma_{\psi}, \Gamma_{\mathrm{A}}, w\right\}$ or any density $\vec{\psi}, \overrightarrow{\tilde{\psi}}, \mathbf{A}, \tilde{\mathbf{A}}$. The static $\zeta$-functions following from (22) and (23) read:

$$
\begin{aligned}
\zeta_{\psi} & =(3-\varsigma) e^{2}, \quad \zeta_{\mathrm{A}}=-n \frac{e^{2}}{6}, \\
\zeta_{r} & =\frac{n+2}{6} u-\varsigma e^{2}, \quad \zeta_{e^{2}}=(3-\varsigma) e^{2}, \\
\zeta_{u} & =\frac{n+8}{6} u-2 \varsigma e^{2}+18 \frac{e^{4}}{u} .
\end{aligned}
$$

The $\zeta$-function of the gauge parameter follows from (17) and (18) as $\zeta_{\varsigma}=0$. The dynamical $\zeta$-functions follow from (24) and (25). They are:

$$
\zeta_{\tilde{\psi}}=-(3-\varsigma) e^{2}-2 e^{2} \varsigma \frac{w}{1+w}, \quad \zeta_{\tilde{\AA}}=n \frac{e^{2}}{2}\left(\frac{1}{3}-\frac{1}{w}\right),
$$

and the $\zeta$-functions for the kinetic coefficients are obtained from the above relations (20) and (21)

$$
\zeta_{\Gamma_{\psi}}=\frac{1}{2}\left(\zeta_{\psi}-\zeta_{\tilde{\psi}}\right), \quad \zeta_{\Gamma_{\mathrm{A}}}=\frac{1}{2}\left(\zeta_{\mathrm{A}}-\zeta_{\tilde{\mathrm{A}}}\right)
$$


Inserting the one loop results (31) they read:

$$
\zeta_{\Gamma_{\psi}}=e^{2}\left(3-\frac{\varsigma}{1+w}\right), \quad \zeta_{\Gamma_{\mathrm{A}}}=-n \frac{e^{2}}{2}\left(\frac{1}{3}-\frac{1}{2 w}\right) .
$$

According to (26) and (32), the $\zeta$-function of the time scale ratio $w$ is then found as follows:

$$
\zeta_{w}=\zeta_{\Gamma_{\psi}}-\zeta_{\Gamma_{\mathrm{A}}}=e^{2}\left(3-\frac{\varsigma}{1+w}+\frac{n}{2}\left(\frac{1}{3}-\frac{1}{2 w}\right)\right) .
$$

\section{Fixed points and exponents}

The behavior of the model parameters under renormalization is described by the flow equations

$$
\ell \frac{\mathrm{d} \alpha_{i}}{\mathrm{~d} \ell}=\beta_{\alpha_{i}}(\{\alpha\}, \varsigma)
$$

where the right hand sides of the equations are determined by appropriate $\beta$-functions (the index $i$ runs over the set of parameters). The $\beta$-functions are generally defined as follows:

$$
\beta_{\alpha_{i}}\left(\left\{\alpha_{i}\right\}, \varsigma\right)=\alpha_{i}\left[-c_{i}-p_{i} \zeta_{\psi}-q_{i} \zeta_{\mathrm{A}}+\zeta_{\alpha_{i}}\right]
$$

The coefficients $c_{i}, p_{i}$ and $q_{i}$ follow from the general renormalization of $\alpha_{i}$ as used above

$$
\stackrel{\circ}{\alpha}_{i}=\kappa^{c_{i}} \mathcal{A}_{d}^{-\left(p_{i}+q_{i}\right) / 2} Z_{\psi}^{-p_{i}} Z_{\mathrm{A}}^{-q_{i}} Z_{\alpha_{i}} \alpha_{i}
$$

The FPs $\left\{\alpha^{\star}\right\}$ are defined by the zeros of the right hand sides of (35). A FP is stable if all stability exponents $\omega_{i}$ are positive. The stability exponents $\omega_{i}$ are defined by the eigenvalues of the matrix $\partial \beta_{\alpha_{i}} /\left.\partial \alpha_{j}\right|_{\{\alpha\}=\left\{\alpha^{\star}\right\}}$.

\subsection{Statics}

The one-loop static $\beta$-functions appear to be independent of the gauge determined by the parameter $\varsigma$ and are the same as in [2]:

$$
\beta_{u}=\left(-\varepsilon u-6 e^{2} u+\frac{n+8}{6} u^{2}+18 e^{4}\right), \quad \beta_{e^{2}}=e^{2}\left(-\varepsilon+\frac{n}{6} e^{2}\right) .
$$

These $\beta$-functions have four FPs: (i) the Gaussian FP with $u^{\star}=e^{\star 2}=0$, (ii) the FP of the uncharged XY-model $\left(u^{\star}=6 \varepsilon /(n+8), e^{\star}=0\right)$, (iii) the tricritical FP with $u^{\star}=0, e^{\star 2}=6 \varepsilon / n$ and (iv) the charged FP with both couplings $u^{\star} \neq 0$ and $e^{\star 2}=6 \varepsilon / n$ nonzero. In one loop order the charged FP exists only for $n>365.9$ [2]. However, in higher loop order and using summation procedures, a charged FP is also found for $n=2$ [4]. In one loop order for $n<365.9$ starting with $e^{2} \neq 0$ and $u \neq 0$, the flow escapes to $e^{2} \rightarrow e^{\star 2}$ and $u \rightarrow \infty$.

Critical exponents are calculated at a stable accessible FP. Expressions for the correlation length critical exponent $\nu$, the specific heat exponent $\alpha$ and the pair correlation function critical exponent $\eta$ are correspondingly:

$$
\begin{aligned}
\nu^{-1} & =2-\zeta_{r}\left(u^{\star}, e^{\star}, \varsigma\right)+\zeta_{\psi}\left(u^{\star}, e^{\star}, \varsigma\right)=2-\frac{n+2}{6} u^{\star}+3 e^{\star 2}, \\
\alpha & =2-d \nu \\
\eta & =\zeta_{\psi}\left(u^{\star}, e^{\star}, \varsigma\right)=(3-\varsigma) e^{\star 2} .
\end{aligned}
$$

All other static critical exponents may be found from scaling relations. Since the FP values $u^{\star}$ and $e^{\star}$ are gauge independent this is also valid for $\nu$ and $\alpha$ although the gauge $\varsigma$ appears in the $\zeta$-functions (39) explicitly. On the contrary, this dependence on the gauge remains in $\eta$ (see (41)). 
All the other exponents become gauge dependent since they are in any case related to $\eta$ by the scaling laws. However, let us note that only at the charged $\mathrm{FP}\left(e^{\star 2} \neq 0\right)$ a gauge dependence enters the $\zeta$-functions. This holds in all orders of the loop expansion due to the structure of the interaction part of the Lagrangian (9).

From renormalization of $\varsigma$ in $(17)$, the flow of the gauge parameter reads

$$
\ell \frac{\mathrm{d} \varsigma}{\mathrm{d} \ell}=\varsigma \zeta_{\mathrm{A}}=\varsigma^{2} \frac{n}{6} e^{2}
$$

reaching zero at the charged FP. This means only that the transverse gauge is invariant under renormalization.

\subsection{Dynamics}

The FP values for the time scale ratio can be found from the zeros of the corresponding $\beta$ function

$$
\beta_{w}=w\left(\zeta_{\Gamma_{\psi}}-\zeta_{\Gamma_{\mathrm{A}}}\right)=e^{2} w\left(3-\varsigma+\varsigma \frac{w}{1+w}+\frac{n}{2}\left(\frac{1}{3}-\frac{1}{2 w}\right)\right) .
$$

These zeros depend in one loop order on the value of the minimal coupling (charge) $e$ at a stable static FP. Only for non-zero charge a specific value for a possible FP is found in this order ( $w$ should be positive)

$$
w^{\star}=\frac{n-12(3-\varsigma)+\sqrt{(n-12(3-\varsigma))^{2}+24 n(18+n)}}{4(18+n)} .
$$

The corresponding stability exponent $\omega_{w}$ :

$$
\omega_{w}=\frac{\partial \beta}{\partial w}=e^{\star 2} w^{\star}\left(\frac{\varsigma}{\left(1+w^{\star}\right)^{2}}+\frac{n}{4 w^{\star 2}}\right),
$$

turns out to be positive and thus the finite FP is stable. Another FP is the infinite FP. In order to find out its stability we introduce

$$
\rho=\frac{w}{1+w}
$$

mapping the range of $w$ into the interval $[0,1]$. The corresponding $\beta$-function reads

$$
\beta_{\rho}=\rho(1-\rho) e^{2}\left(3-\varsigma+\varsigma \rho+\frac{n}{2}\left(\frac{1}{3}-\frac{1-\rho}{2 \rho}\right)\right)=(1-\rho) e^{2}\left((3-\varsigma) \rho+\varsigma \rho^{2}+5 \frac{n}{12} \rho-\frac{n}{4}\right) .
$$

However, the $\mathrm{FP} \rho^{\star}=1$ corresponding to the infinite $\mathrm{FP} w^{\star-1}=0$ is unstable with the stability exponent $\omega_{\rho}=-(1+18 / n) \varepsilon$.

The dynamical critical exponents for the OP and the GF are calculated from

$$
z_{\psi}=2+\zeta_{\Gamma_{\psi}}\left(u^{\star}, e^{\star}, w^{\star}, \varsigma\right), \quad z_{\mathrm{A}}=2+\zeta_{\Gamma_{\mathrm{A}}}\left(u^{\star}, e^{\star}, w^{\star}\right)
$$

Whereas an explicit gauge dependence is found in $\zeta_{\Gamma_{\psi}}$ this is not the case for $\zeta_{\Gamma_{\mathrm{A}}}$, at least in one loop order. This property is expected to hold in all orders. In general at the charged FP $\left(e^{\star 2}=6 \varepsilon / n\right)$ the exponents take the values

$$
\begin{aligned}
z_{\psi} & =2+\frac{18}{n} \varepsilon-\varsigma \frac{6}{n} \frac{\varepsilon}{1+w^{\star}} \\
z_{\mathrm{A}} & =2-\varepsilon+\frac{3 \varepsilon}{2 w^{\star}}
\end{aligned}
$$

Since $w^{\star}$ depends on the gauge for finite FP, the dynamical exponent of the GF, $z_{\mathrm{A}}$, depends on the gauge in contradiction to the physical requirement that observable quantities should be gauge 
independent. Only in the case of infinite FP a gauge independent value is possible. Then the OP exponent would be finite:

$$
z_{\psi}=2+\frac{18}{n} \varepsilon
$$

as well as independent of the gauge but different from $z_{\mathrm{A}}$. This result for $z_{\psi}$ has already been obtained in another context $[22,23]$, where the dynamics of the Bose field OP has been considered by taking into account the renormalization of the Matsubara frequency term in the static functional (3). In this way quantum correlations can be taken into account. The model considered here is purely classical and the result is the consequence of the gauge field relaxing much faster than the $\mathrm{OP}\left(w^{\star} \rightarrow \infty\right)$. However, the infinite FP is not stable in one loop order. It is dynamically unstable for all $n$.

It should be noted that for the gauge $\varsigma=1$, the results of [17] are reproducible. It should also be remarked that in one loop order the calculation of the field theoretic functions is simpler in the transverse gauge $(\varsigma=0)$ due to the observation that less graphical contributions in the loop expansion are nonzero [9]. To our knowledge, the gauge independence of the static FP values for the minimal coupling and fourth order coupling has not been proven. As it has been shown here the finite $\mathrm{FP}$ value of the dynamical time scale $w$ is already gauge dependent in one loop order, which leads to a gauge dependent critical dynamical exponent of the GF. In our opinion, this would hold for every order for the finite nonzero $\mathrm{FP}$ value $w^{\star}$. At the uncharged $\mathrm{FP}$, both dynamical exponents would be independent of the gauge.

\section{Conclusion and outlook}

For the dynamical model suggested by Lannert, Vishveshwara and Fisher [17] we have demonstrated that the OP dynamical exponent is gauge dependent. Therefore, the exponent for the divergence of the electric conductivity is also gauge dependent at the stable strong scaling FP where the dynamical critical exponents of the $\mathrm{OP}, z_{\psi}$, and the GF, $z_{\mathrm{A}}$, are the same. The only way out in a higher loop order calculation is for the stability of the strong scaling FP to be changed and for the infinite weak scaling FP to become a stable one. As one knows from other examples, one loop order calculations may not be conclusive and lead to the results which have to be taken with care. In higher order perturbation expansion, the stability of the FPs may be changed. A two loop calculation may clarify the situation. Recent progress [24] in the nonperturbative version of dynamical renormalization theory applied to this more complicated model would be also worthwhile.

\section{Acknowledgements}

This work was supported by Fonds zur Förderung der wissenschaftlichen Forschung under Project No. P16574. We thank one of the referees bringing [22] to our knowledge.

\section{Appendix}

\section{A. Diagrams for the one loop order calculation}

Expressions for vertex functions are calculated using the Feynman diagrams. They are constructed from the corresponding propagators and vertices. In our case the propagators are described by Gaussian part $\mathcal{L}_{0}(8)$ of Lagrangian and shown in figure 1. Response propagators $G(\bar{k})$ and $H(\bar{k})$ are equal to

$$
G(\bar{k})=\frac{1}{\left(-\mathrm{i} \omega+\Gamma_{\psi}^{\circ}\left(\stackrel{\circ}{r}+k^{2}\right)\right)} \quad \text { and } \quad H(\bar{k})=\frac{P_{\alpha \beta}}{\left(-\mathrm{i} \omega+\stackrel{\circ}{\Gamma}_{\mathrm{A}} k^{2}\right)}+\frac{\stackrel{\circ}{\varsigma}\left(\delta_{\alpha \beta}-P_{\alpha \beta}\right)}{\left(-\mathrm{i} \omega \stackrel{\circ}{\varsigma}+\stackrel{\circ}{\Gamma}_{\mathrm{A}} k^{2}\right)},
$$




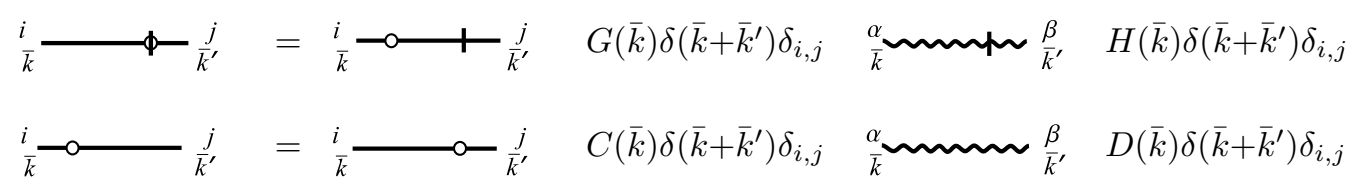

Figure 1. Propagators for constructing Feynman graphs. $G(\bar{k})$ are response propagators for $\left\langle\psi_{i}(\bar{k}) \tilde{\psi}_{j}^{+}\left(\bar{k}^{\prime}\right)\right\rangle$ and $\left\langle\psi_{i}^{+}(\bar{k}) \tilde{\psi}_{j}\left(\bar{k}^{\prime}\right)\right\rangle$ (solid line), $H(\bar{k})$ are response propagators for $\left\langle A_{\alpha}(\bar{k}) \tilde{A}_{\beta}\left(\bar{k}^{\prime}\right)\right\rangle$ (wavy line), while $C(\bar{k})$ and $D(\bar{k})$ are correlation propagators. As small circle ' $\circ$ ' in the figure denotes complex conjugated field $\psi^{+}$, while a small vertical line '|' denotes the auxiliary fields $\tilde{\psi}$ and $\tilde{\boldsymbol{A}}$.

while the correlation propagators $C(\bar{k})$ and $D(\bar{k})$ are equal to

$$
C(\bar{k})=\frac{2 \stackrel{\circ}{\Gamma}}{\left|-\mathrm{i} \omega+\stackrel{\circ}{\Gamma}\left(\stackrel{\circ}{r}+k^{2}\right)\right|^{2}} \quad \text { and } \quad D(\bar{k})=\frac{2 \stackrel{\circ}{\Gamma}_{\mathrm{A}} k^{2} P_{\alpha \beta}}{\left|-\mathrm{i} \omega+\stackrel{\circ}{\Gamma}_{\mathrm{A}} k^{2}\right|^{2}}+\frac{\stackrel{\circ}{\varsigma} 2^{2} 2 \stackrel{\circ}{\mathrm{A}}_{\mathrm{A}} k^{2}\left(\delta_{\alpha \beta}-P_{\alpha \beta}\right)}{\left|-\mathrm{i} \omega \stackrel{\circ}{\varsigma}+\stackrel{\circ}{\Gamma}_{\mathrm{A}} k^{2}\right|^{2}},
$$

where $P_{\alpha \beta}$ is projection operator $P_{\alpha \beta}=\delta_{\alpha \beta}-k_{\alpha} k_{\beta} / k^{2}$. In the above equations, we use the notation $\bar{k}=(\vec{k}, \omega)$.

The vertices described by interaction part $\mathcal{L}_{1}(9)$ of Lagrangian are presented in figure 2.

a

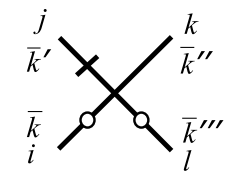

$\stackrel{\circ}{\Gamma}_{\psi} X \delta\left(\bar{k}+\bar{k}^{\prime}+\bar{k}^{\prime \prime}+\bar{k}^{\prime \prime \prime}\right)$

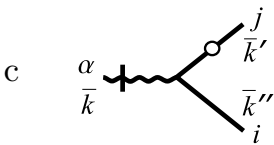

$\stackrel{\circ}{\Gamma}_{\mathrm{A}} \stackrel{\circ}{ } / 2\left(k_{\alpha}^{\prime}-k_{\alpha}^{\prime \prime}\right) \delta\left(\bar{k}+\bar{k}^{\prime}+\bar{k}^{\prime \prime}\right) \delta_{i j}$

f

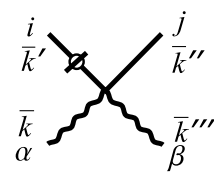

$\stackrel{\circ}{\Gamma}_{\psi} \stackrel{\circ}{2}^{2} \delta\left(\bar{k}+\bar{k}^{\prime}+\bar{k}^{\prime \prime}\right) \delta_{i j} \delta_{\alpha \beta}$ $\mathrm{b}$

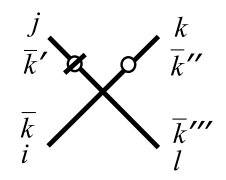

$\stackrel{\circ}{\Gamma}_{\psi} X \delta\left(\bar{k}+\bar{k}^{\prime}+\bar{k}^{\prime \prime}+\bar{k}^{\prime \prime \prime}\right)$

d

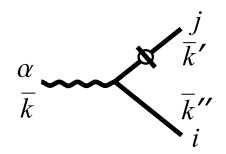

$-2 \stackrel{\circ}{\Gamma}_{\psi} \stackrel{\circ}{k_{\alpha}^{\prime \prime}} \delta\left(\bar{k}+\bar{k}^{\prime}+\bar{k}^{\prime \prime}\right) \delta_{i j}$

g

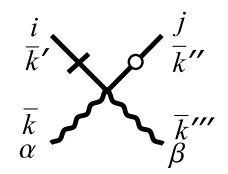

$\mathrm{h}$

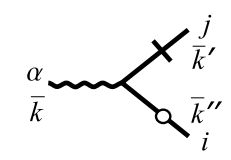

$2 \Gamma_{\psi}^{\circ} \stackrel{\circ}{e} k_{\alpha}^{\prime \prime} \delta\left(\bar{k}+\bar{k}^{\prime}+\bar{k}^{\prime \prime}\right) \delta_{i j}$

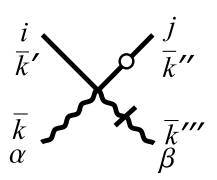

$\stackrel{\circ}{\Gamma}_{\psi} e^{2} \delta\left(\bar{k}+\bar{k}^{\prime}+\bar{k}^{\prime \prime}\right) \delta_{i j} \delta_{\alpha \beta}$

Figure 2. Vertices for our model. In vertices $\mathbf{a}$ and $\mathbf{b}$ notation $X$ is equal $\grave{u} / 3 !\left(\delta_{i, j} \delta_{k, l}+\delta_{i, l} \delta_{j, k}\right) / 2$.

The one-loop diagrams for vertex functions $\stackrel{\circ}{\Gamma}_{\psi \tilde{\psi}^{+}}$and $\stackrel{\circ}{\Gamma}_{A \tilde{A}}$ are depicted in figure 3 . 

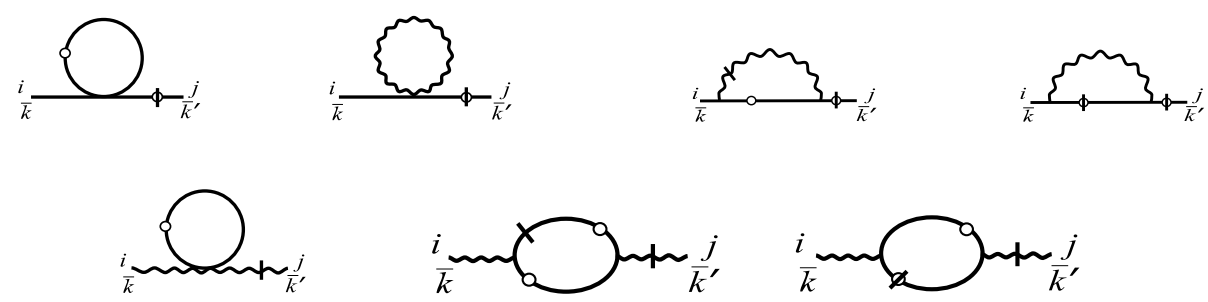

Figure 3. One-loop contributions to the vertex functions $\stackrel{\circ}{\Gamma}_{\psi \tilde{\psi}^{+}}$(first line) and $\stackrel{\circ}{\Gamma}_{A \tilde{A}}$ (second line).

\section{References}

1. Lobb J. Phys. Rev. B, 1987, 36, 3930.

2. Halperin B.I., Lubensky T.C., Ma S.K. Phys. Rev. Lett., 1974, 32, 292.

3. Kolnberger S., Folk R. Phys. Rev. B, 1990, 41, 4083.

4. Folk R., Holovatch Yu. J. Phys. A: Math. Gen., 1996, 29, 3409; Folk R., Holovatch Yu. - In: Correlations, Coherence, and Order, ed. by Shopova D.V., Uzunov D.I. Kluwer Academic/Plenum Publishers, N.Y.-London, 1999, 83-116; cond-mat/9807421.

5. Kleinert H. Lett. Nuovo Cimento, 1982, 35, 405; Kiometzis M., Kleinert H., Schakel A.M.J. Phys. Rev. Lett., 1994, 73, 1975; Fortschr. Phys., 1995, 43, 697.

6. Dasgupta C., Halperin B.I. Phys. Rev. Lett., 1981, 47, 1556; Bartolomew J. Phys. Rev. B, 1983, 28, 5378.

7. Schneider T., Khasanov R., Conder K., Pomjakushina E., Bruetsch R., Keller H. J. Phys. Condens. Matter, 2004, 16, L437.

8. Schneider T., Khasanov R., Keller H. Phys. Rev. Lett., 2005, 94, 077002.

9. Kang J.S. Phys. Rev. D, 1974, 10, 3455.

10. Kleinert H., Schakel A.M.J. Phys. Rev. Lett., 2003, 90, 097001.

11. Aji V., Goldenfeld N. Phys. Rev. Lett., 2001, 87, 197003.

12. Folk R., Moser G. J. Phys. A: Math. Gen., 2006, 39, R207-R313.

13. Lidmar J., Wallin M., Wengel C., Girvin S.M., Young A.P. Phys. Rev. B, 1998, 58, 2827.

14. Nogueira F.S., Manke D. Phys. Rev. B, 2005, 72, 014541.

15. Dzyaloshinskii I.E., Volovick G.E. Ann. Phys. (NY), 1980, 125, 67.

16. see the comments by Lidmar and the answer by Aji and Goldenfeld in Phys. Rev. Lett., 2002, 89, 109701, 109702, respectively.

17. Lannert C., Vishveshwara S., Fisher M.P.A. Phys. Rev. Lett., 2004, 92, 097004.

18. In statics a difference in the scaling of lengths appears depending on which FP is stable. At the XY FP (FP value of the coupling to the GF is zero) the OP correlation length $\xi$ scales with the exponent $\nu$, whereas the penetration length $\lambda$ with $\nu / 2$. At the charged FP (FP value of the coupling to the GF is non-zero) both lengths scale with the exponent $\nu$ (see [20]).

19. De Dominicis C., Peliti L. Phys. Rev. B, 1978, 18, 353; Dohm V. Phys. Rev. B, 1991, 44, 2697; Err., ibid., 2006, 73, 09990(E).

20. Herbut I.F., Tešanovic Z. Phys. Rev. Lett., 1996, 76, 4588.

21. Bausch R., Janssen H.K., Wagner H. Z. Phys. B, 1976, 24, 113.

22. Bushev M.K., Uzunov D.I. Phys. Lett. A, 1980, 76, 306; Err., ibid., 1980, 78, 491.

23. For a discussion see also Shopova D.V., Uzunov D.I. Fluctuation and gauge effects on the critical behavior of superconductors, preprint cond-mat/0701266.

24. Canet L., Chate H. Non-perturbative Approach to Critical Dynamics, preprint cond-mat/0610468, 2006. 


\title{
Калібрувальна залежність критичної динаміки надпровідного переходу
}

\author{
М.Дудка ${ }^{1,2}$, Р.Фольк, ${ }^{2}$, Г.Мозер ${ }^{3}$
}

1 Інститут фізики конденсованих систем, Національна академія наук України, UA-79011 Львів, Україна

2 Інститут теоретичної фізики Університету Йогана Кеплера міста Лінц, А-4040 Лінц, Австрія

3 Інститут фізики і біофізики Університету міста Зальцбург, А-5020 Зальцбург, Австрія

Отримано 27 грудня 2006 р., в остаточному вигляді - 7 травня 2007 р.

Переглядається динаміка надпровідників в зарядженому режимі в межах теорії поля. Запропонована раніше [Lannert et al. Phys. Rev. Lett. 92, 097004 (2004)] модель типу Гінзбурга-Ландау з комплексним параметром порядку зв'язаним з калібрувальним полем використовується для дослідження динаміки. Приймаючи релаксаційну динаміку для обох величин, ренорм-групові функції обчислюються в рамках однопетлевого наближення для різних виборів калібрування. Калібрувально незалежні результати для розбіжності вимірюваної електричної провідності отримуються тільки в нерухомій точці слабкого скейлінгу, нестійкій в однопелевому порядку, де часові масштаби параметра порядку та калібрувального поля відрізняються.

Ключові слова: критична динаміка, надпровідний перехід

PACS: 05.70.Jk, 64.60.Ak, 64.60.Ht, 74.20.-z 